\title{
Passaging and cryopreservation during culture of human pluripotent stem cells
}

\author{
Woo Jung Ho* and Hea-Jo Yoon \\ Stem Cell Research Center, Apgujeong Miracle Clinic, South Korea
}

\section{Culture period and number of passaging}

It is important to maintain stem cells in an undifferentiated state if the undifferentiated stem cells are to be used for regenerative therapy. However, during long culture periods, stem cell karyotypic aberrations may be present to maintain self-renewing cells in a stable state, particularly exposure to new culture conditions increases the likelihood of malignant transformation [1]. The WHO guidelines in 2013 recommended to passage cells for a maximum of 15 passages or a maximum of 3 months [2]. However, limitation of passage numbers and duration of passaging can vary depending on the state of the cells, the method and quality of passage, and the split ratio.

\section{Use master stock to reduce numbers of passaging}

HPSCs should be cryopreserved if they are judged to be in a stable state in order to reduce differentiation, genetic instability, contamination and cross contamination caused by continued passage [2]. Small-scale cryopreserved seed cell banks can supply stable cells with yet low passage for research and treatment over many years. After thawing, cell viability (eg, MTT, Alamar Blue, intracellular ATP assay, phenol red) and growth rate (eg, proliferation rate by cell counting) can be measured.

\section{Passaging}

Passaging (subculture) means transferring cells from one culture vessel to one or more new culture vessels to prevent overcrowding. Passaging is used to decrease the detrimental effects of overcrowding and expand the culture. Usually, when the cultured cells reaches about $70-85 \%$ of the cultured surface area, passaging is made, but confluency (the ratio of cells to the culture surface area) measurement may vary depending on the experimenter [2].

Cell-to-cell and cell-substrate attachment are mediated by cell surface receptors and extracellular matrix proteins. $\mathrm{Ca}^{2+}$ ion, proteoglycan from cells and serum are important in this process [3]. Therefore, protein degradation and $\mathrm{Ca}^{2+}$ chelation is often required for passaging. Cells with weak adhesion could be detached by treatment with trypsin and shaking. However, strongly adherent cells should be treated with strong proteases such as pronase, dispase, and collagenase.

The methods of detachment depend on culture conditions (e.g. growth medium, surface matrix), the cell grade (e.g., research, therapy), and current state of the culture (e.g. high/low passage, extent of differentiation) [2]. Cell detachment methods include chemical, enzymatic treatment, and mechanical methods [4]. Using enzymes for detachment is the least time consuming, but it is likely to increase the genetic instability of stem cells. Mechanical passaging can be used when the efficiency of the passing (rate of attachment after passage) is not important or the differentiation is good.

Positive selection and negative selection: Since some of the stem cells can be differentiated, there are two ways to separate the undifferentiated stem cells from differentiated stem cells. Positive selection is a method of passaging undifferentiated cells by manually dissecting them, and negative selection is a method of selectively removing the differentiated cells when colonies are relatively small and there are areas of excessive differentiation [2].

\section{Chemical or enzymatic passaging}

The choice of reagent used for passaging depends on the matrix used to support the cells and what grade of manufacture is desired. Human pluripotent stem cells (HPSCs) cultivated on mouse embryo fibroblasts (MEFs) are typically dissociated with collagenase or trypsin. EDTA and dispase are used to splitting colonies as clumps and trypsin is used to make a single cell suspension. EDTA does not usually require scraping, but dispases require direct contact with the cells.

Rho-associated, coiled-coil containing protein kinase (ROCK) inhibitors can be used to reduce the number of cells with attachment failure after passage because of loss of cell viability [4]. However, the effect of ROCK inhibitors on long-term passaging was not revealed.

The number of times a culture is passaged should be recorded with the split ratio and cell doubling time to estimate the relative age of cells cultured. High-passage cells generally double robustly but are more likely to acquire genetic abnormalities while adapting to ex vitro conditions. Low-passage cells can exhibit unpredictable growth rates and spontaneous differentiation potential [5]. Plating efficiency (the ability of cells to attach and divide efficiently) is influenced by parameters including clump size after passaging, split ratio, strength of mechanical force used to break up the cell clumps, and growth conditions [2]. Determining the proper split ratio is particularly important for low passage cells and an inappropriately high split ratio can affect cell recovery, adhesion, and genomic stability after splitting.

\section{Adaptation to new culture conditions}

The adaptation to new culture environment and post-adaptation growth rates can be influenced by changes in culture conditions (e.g.

${ }^{\star}$ Correspondence to: Woo Jung Ho MD, Stem Cell Research Center, Apgujeong Miracle Clinic, Seoul, South Korea, E-mail: miracleps77@naver.com

Received: March 02, 2019; Accepted: March 18, 2019; Published: March 20 2019 
MEF co-culture to feeder free culture), inherent property of the cells themselves, and cell density after splitting.

\section{Cryopreservation}

To preserve cell function for an extended period of time, cells are diluted with cryoprotectant solution before freezing, and stored by cryopreservation. Cryopreservation should be performed carefully considering cryoprotectants, additives, cooling rate, storage conditions, or cell function recovery methods. Prior to cryopreservation, cells should have low levels of differentiation, high viability, and optimal growth rate (i.e., exponential phase of growth curve) [2].

\section{Methods of cryopreservation}

Slow freezing and rapid thawing methods using cryoprotectants such as $10 \%$ DMSO are generally used for cryopreservation. There is a method of storing vials containing stem cells overnight in a freezer at $-80^{\circ} \mathrm{C}$ and then transferring them to a liquid nitrogen (LN2) tank. Alternatives are cooling to a desired temperature precisely at a cooling rate of 0.3 to $5^{\circ} \mathrm{C}$ per minute using a controlled-rate freezer [6]. Stem cells can be cryopreservated using vitrification (i.e. solidification without crystal ice formation), which rapidly freezes under high concentrations of cryoprotectants [7]. If done properly, either slow or rapid freezing will result in successful cryopreservation.

\section{Cryoprotective agent (CPA)}

A cryopreservative acts to reduce the formation of ice by increasing the total concentration of solutes. To be biologically effective, it must be able to penetrate into cells and low toxicity should be required [8]. Diffusion and osmosis therefore have an important influence on the application of cryoprotectants, removal of cryoprotectants, freezing processes and thawing [8].

\section{Storage: Liquid nitrogen (LN2)}

Liquid nitrogen is a nitrogen gas present in liquid form at $-196^{\circ} \mathrm{C}$. The hazards arising from LN2 are related to temperature or volume expansion associated with gas changes. Skin contact with liquid nitrogen can cause tissue damage due to frostbite. Liquid nitrogen changes to gas at room temperature and expands 700 times, resulting in hypoxia or explosion of container. Vapor-phase liquid nitrogen used for cryopreservation minimizes the risk of cross-contamination and the risk associated with pressure buildup [9]. Cells stored at $-80^{\circ} \mathrm{C}$ lose their viability through devitrification. Therefore storage at $-80^{\circ} \mathrm{C}$ should only be used for short-term storage or transport [2].

\section{Cell function recovery after thawing}

After thawing of cryopreserved cells, the cell's ability to regenerate is very variable, and stable recovery (increase in cell viability) is possible by controlling the temperature rise for thawing at a constant rate [6]. Thawing into different culture medium and/or matrix with other cultures can affect growth kinetics and stability. For example, some stem cells may have unexpected results when they changed from the presence feeder layers to feeder-free conditions through thawing. When evaluating the recovery of cells after thawing, it is necessary to observe the state of the cells for a long period of time. An immediate assessment at post-thaw may lead to false conclusions about the integrity of the cells [2].

\section{References}

1. Lincoln CK, Gabridge MG (1998) Cell culture contamination: sources, consequences, prevention, and elimination. Methods in cell biology, Academic Press 49-65. [Crossref]

2. Pamies D, Bal-Price A, Simeonov A, Tagle D, Allen D, et al. (2017) Good cell culture practice for stem cells and stem-cell-derived models. Alternatives to Animal Experimentation: ALTEX 34: 95-132. [Crossref]

3. Frantz C, Stewart KM, Weaver VM (2010) The extracellular matrix at a glance. $J$ Cell Sci 123: 4195-4200. [Crossref]

4. Beers J, Gulbranson DR, George N, Siniscalchi LI, Jones J, et al. (2012) Passaging and colony expansion of human pluripotent stem cells by enzyme-free dissociation in chemically defined culture conditions. Nature Protocols 7: 2029. [Crossref]

5. Pamies D, Bal-Price A, Chesne C, Coecke S, Dinnyes A, et al. (2018) Advanced Good Cell Culture Practice for human primary, stem cell-derived and organoid models as well as microphysiological systems. Altex 35: 353-378. [Crossref]

6. Ware CB, Nelson AM, Anthony Blau C (2005) Controlled-rate freezing of human ES cells. Biotechniques 38: 879-883. [Crossref]

7. Vajta G, Kuwayama M (2008) Improving cryopreservation systems. Theriogenology 65: 236-244. [Crossref]

8. Benson EE Cryopreservation theory. Plant cryopreservation: A practical guide Springer 15-32.

9. Hay RJ (1978) Preservation of cell-culture stocks in liquid nitrogen. Methods in Cell Science 4: 787-790.

Copyright: (C2019 Ho WJ. This is an open-access article distributed under the terms of the Creative Commons Attribution License, which permits unrestricted use, distribution, and reproduction in any medium, provided the original author and source are credited. 